\title{
Equations Of State of Hydrogen-Helium and Carbon-Oxygen Mixtures
}

\author{
P. Godon, G. Shaviv, J. Ashkenazi* and A. Kovetz** \\ Department of Physics, Technion-Israel Institute of Technology 32000 Haifa, Israel \\ * Department of Physics, University of Miami, Coral Gables, FL 33124, U.S.A. \\ ** Department of Physics and Astronomy and Department of Enviornmental Science \\ Tel-Aviv University, Ramat Aviv, Israel
}

The usual approach to the problem of the Equations Of State (EOS) for White Dwarfs and super giant Planets, under the conditions of high densities and low temperatures, faces two problems.

The first has to do with Pressure Ionization : the energy levels of the electrons in the case of extreme pressure ionization cannot be treated in the same way as the energy levels in a single isolated atom. The simple treatment of pressure ionization, in which the level of the continuum is reduced by an amount equal to the electrostatic energy, leads to problem because the number of energy levels, entering the Saha equation (partition functions), is not conserved, also the usual approach may lead to absurd results in which ions recombine as the density increases.

The second problem is the question of the microscopic separation of species in a mixture : is the prefered state a mixed crystal of two species or two separeted crystals (one of each specy) ?

The EOS in Astrophysics treats the electrons as a gas of free particles (1) (plane waves function) obeing the Fermi-Dirac statistics, where the ionic background forms a crystaline structure. At zero temperature the energy of the ions is then given by the Zero Point Oscillations (ZPO) (2) energy. At finite temperatures the phonons spectrum of the lattice (quasiharmonic approximation) is added to it.

Another approach to the problem of the EOS is by means of Solid State methods. The electrons individual energy levels form energy bands which take into account the conservation of the number of energy levels, and their wave function is a Bloch sum of a linear combinations of atomic orbitals (LCAO). The ions are sup- 
posed to be fixed in the crystal lattice (Adiabatic approximation).

Since the astrophysical approach to the EOS cannot answer the above two questions, we resort to the solid state approach. In this contribution we report mainly on the results for the second problem.

The method we used in this work is the Linear Muffin-Tin Orbitals (LMTO) (3) method in which the wave functions are Bloch sums of Linear Orbitals defined by a Muffin-Tin (MT) potential. The MT potential is spherically symetric in spheres around the ions sites and of constant value in the interstitial regions. Then a variational method is used to derive the correct energy bands, their width, the density of state, etc...

It is of great interest to compare the results for the EOS obtained in the two methods. Such a comparison is shown in figure 1 and 2 for $\mathrm{H} / \mathrm{He}$. We note that the two methods yield the same EOS to within the accuracy of the methods (less than one percent).

We now turn to the second problem posed.

To answer the question of whether a $\mathrm{H} / \mathrm{He}$ (or a $\mathrm{C} / \mathrm{O}$ ) mixture separates or not, we consider a volume $V_{*}$ of the star, under pressure $P_{*}$ and temperature $T_{*}$. We suppose that in this volume separated crystals of $\mathrm{H}$ and $\mathrm{He}$ have been formed. Let index 1 denote Hydrogen and 2 Helium. Then $V_{*}=V_{1}+V_{2}, N=N_{1}+N_{2}$ (number of particles in $V_{*}$ ). The most probable state for the coexistence of the two crystals in volume $V_{*}$ is obtained by minimizing the free energy. This leads to the condition :

$P_{1}=P_{2} \quad ; \mu_{1}=\mu_{2}$.

Where $P_{i}$ is the pressure and $\mu_{i}$ the chemical potential $(i=1,2)$. Let denote $P=P_{1}=P_{2}$.

If $P \leq P_{*}$ then the pressure inside the volume $V_{*}$ is not high enough to avoid separation of the mixed crystal. If $P \geq P_{*}$ then the separation process is stopped by the pressure inside $V_{*}$.

The results obtained, within the accuracy of the LMTO calculation (one percent), shows that the pressures $P_{*}$ and $P$ were equal. This leads us to conclude that there is no prefered state between a mixed crystal of two species and two separated crystals on a microscopic scale : microscopic separation does not require energy. Consequently the separation problem is a macroscopic problem that has to be treated within the frame work of the evolution of the star. 


\section{References}

(1) A. Kovetz and G. Shaviv, Astron. Astrophys. 8, 398-403 (1970).

G. Shaviv and A. Kovetz, Astron. Astrophys. 16, $72-76$ (1972).

(2) E. E. Salpeter, Astrophys. J., 134, 669 (1961).

(3) O. K. Andersen, Phys. Rev. B12, 3060 (1975).

H. L. Skriver, The LMTO Method, Springer Verlag (1984).

\section{Comparison between LMTO and EVOLVE}

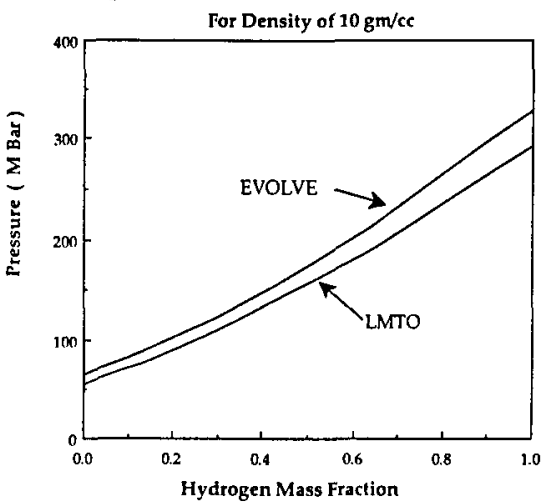

Fig.l Comparison between the Astrophysical (Evolve) and the solid state (LMTO) results for the pressure. We have omitted the ionic contribution (ZPO) in the LMTO (while it is included in Evolve).

Comparison Between Evolve and LMTO For Density of $100 \mathrm{gm} / \mathrm{cc}$

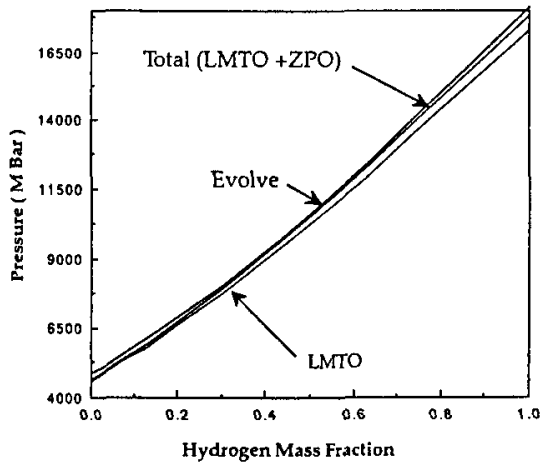

Fig. 2 H-He mixture for a density of $100 \mathrm{gm} / \mathrm{CC}$ and $\mathrm{T}=0$. We compare between the full EOS (ions + electrons) obtained by the two different methods. We see that the astrophysical results are good within a few percent.

LMTO Total pressure For density of $100 \mathrm{gm} / \mathrm{cc}$

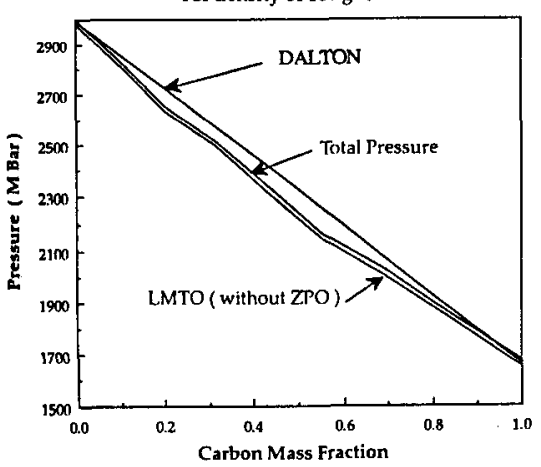

Fig.3 LMTO pressure for a C-O mixture, $\rho=100 \mathrm{gm} / \mathrm{cc}$. 